\title{
THE TOXIC EFFECTS OF ARSENOBENZOL TREATMENT AND THEIR PREVENTION
}

\author{
By DAVID LEES, D.S.O., F.R.C.S. (Ed.).
}

From the earliest days in Igog of the use of Ehrlich's salvarsan in human therapy by Alt and his co-workers toxic effects have been recorded.

The toxic effects produced by the intravenous or intramusclar use of arsenobenzol and its derivatives manifest themselves in many ways, and many factors enter into their causation. They are influenced by-

(I) The health of the individual patient prior to administration of the drug.

(2) The preparation of the patient for this form of treatment.

(3) The drug and the method of preparation of the solution to be used.

(4) The technique of its administration.

(I) The Health of the Individual Patient.-Early in the history of salvarsan Ehrlich drew attention to the fact that toxic effects occurred most frequently in cachectic patients and in those who, in addition to syphilis, had other pathological complications. Diabetes, Addison's disease, advanced cases of nephritis unless of syphilitic origin, gross cardiac disease, and hepatic disease are all conditions in which it is inadvisable to exhibit arsenobenzol treatment. Cases in which the drug should be given guardedly are those with advanced cardio-vascular disease, those with gross syphilitic disease of the central nervous system, cases of syphilitic nephritis or hepatitis, cases of tuberculous or other debilitating disease, and all alcoholics. Apart from these it is always advisable that the general health of the patient should be attended to.

2. The Preparation of the Patient.-In addition to ensuring that the skin, the bowel and the other excretory organs are in good working order it is essential that the patient should not partake of food for at least three hours prior to intravenous treatment by arsenobenzol. Too long fasting, on the other hand, is prone to give rise to 


\section{EFFECTS OF ARSENOBENZOL TREATMENT}

toxic effects. In addition, it is essential that the patient should not indulge in violent exertion for some hours before and after the intravenous administration of the drug. The latter is almost as important as the former in preventing side effects. Abstinence from alcohol, regular habits and daily evacuation of the bowels are other important factors in safeguarding the patient.

3. The Drug and the Method of Preparation of the Solution to be used.-The " 606 " is undoubtedly more toxic than the "9I4" series. In the former great care must be exercised in the proper alkalisation to reduce the mono-sodium to the di-sodium salt. Over-alkalisation, in addition, has a destructive action on the vessel walls. In diluting the drug a minimum of 25 c.c. of the solvent to each 0.I of the "606" drug is essential. Dilution of the drug is not of so great importance in preparing the " $9 \mathrm{I} 4$ " group apart from the fact that it makes for its slower administration. This slowness of administration into the circulation with either " 606 " or " $9 \mathrm{I} 4$ " is one of the most potent factors in avoiding subsequent intolerance. Of equal importance is the avoidance of shaking the solution of the drug. The importance of this has been stressed by Schamberg, ${ }^{1}$ Kolmer and Raiziss, while the same workers and Weiss ${ }^{2}$ state that cloudy or turbid solutions will almost invariably give rise to toxic reactions. An additional factor of importance in avoiding toxic reactions is filtration of the drug to make certain that no undissolved particles are introduced into the system. The distilled water and saline solution used as solvents must be beyond reproach, and in no case, either for intravenous or intramuscular injection, must the solution be kept for any length of time prior to its administration.

4. The Technique of its Administration.-The commoner accidents of the intravenous administration of salvarsan are due to faulty technique, such as a leakage of the drug into the cellular tissues around the vein or, in intramuscular therapy, a deposit of the solution in close proximity to a nerve fibre. The more simple the technique the less fear is there on the part of the patient, and the less likely is the occurrence of some of the more immediate reactions, which are undoubtedly psychic in character. If the accommodation will allow it, the lying posture is always preferable in intravenous therapy to the sitting one, and is much more advantageous for the treatment of any 


\section{BRITISH JOURNAL OF VENEREAL DISEASES}

immediate reaction that may occur. Absolute cleanliness, asepsis and painlessness are essential if the treatment is to be successful, especially in a nervous patient. There are certain patients who, in spite of every care in their preparation and every care in the preparation of the drug and in the technique of its administration, will react badly to its administration. All such immediate reactions can largely be avoided by administering the drug intramuscularly, a comparatively safe and efficient method of giving it. The technique of intramuscular or deep subcutaneous injections, however, requires, if it is to be painless, as much care and skill as that of intravenous medication.

Even when those four contributory factors are carefully guarded against there still are cases in which toxic effects are seen, and from the ætiological point of view there is wide divergence of opinion as to whether the remedy or the disease is the chief causal factor in the production of many of the pathological conditions occurring during and subsequent to the administration of arsenobenzol and its derivatives.

The Nature of the Toxic Reactions which occur during and subsequent to Arsenobenzol Administration.-They may be divided into-

(I) Immediate Reactions.-(a) During the actual giving of the drug ; or $(b)$ within the first twenty-four hours.

(2) Later reactions varying in their onset from a few days up to several months.

(I) (a) In this class may be included headache, malaise, vomiting, rigors, fainting attacks, syncope, palpitation and dyspnœea, transient pain in the gums and a peculiar sense of taste or smell in the mouth, and, chief of all, that group of gross vaso-motor symptoms which are known as nitritoid crises or vaso-dilator reactions.

(I) (b) Within the first twenty-four hours the toxic effects may give rise to temporary headaches and recurrent rigors, attacks of vomiting and diarrhœa, transient albuminuria, herpes, stomatitis and gastro-enteritis, transient skin eruptions either urticarial or erythematous in nature, coryza and conjunctival injection, and general œdema of the face and hands. Delayed vaso-dilator reactions may come on during the first twenty-four hours also, while the condition of serous apoplexy, to which the name hæmorrhagic encephalitis has been applied, gene- 


\section{EFFECTS OF ARSENOBENZOL TREATMENT}

rally has its onset twenty-four to forty-eight hours subsequent to a second or third injection of arsenobenzol or its derivatives.

(2) Later Reactions.-Malaise, general depression, loss of weight, stomatitis and gastro-intestinal disorders, albuminuria, and other signs of toxic actions on the kidney may come on at any time. More serious complications are jaundice, aplastic anæmia and erythema leading on to exfoliative dermatitis.

Early Reactions.-The greater number of these reactions are due to faulty preparation of the patient, fear on the part of the patient, too great concentration of the drug and too rapid administration of the drug. All of them are transient and are rapidly amenable to treatment. Of much more importance are the vaso-dilator reactions. The literature on this subject is enormous, and I shall only briefly refer to a few of the theories concerning their causation. They have been called nitritoid or anaphylactoid because of the similarity of the symptomsrespiratory and cardiac distress, flushing of the face, lachrymation and swelling of the lips, tongue and faceto those seen in anaphylactoid shock. There is no consensus of opinion as to the actual cause of the reaction, but in all cases there is capillary dilatation and damage to the endothelium of the vessel walls. Stokes ${ }^{3}$ is one of the leading advocates of the anaphylactic theory, and is of opinion that the reaction depends on the formation of a precipitate of the drug in the blood stream. This theory is supported by the fact that batches of the drug which are difficult to dissolve, or a drug given in too concentrated a form or too rapidly, are very apt to provoke this vasodilator reaction. Jobling, Peterson and others contend that the proteins of the serum of the patient are the cause or source of the trouble, in other words the anaphylaxis is produced by a change in dispersion of the colloids in the blood stream and is analogous to the change of fibrinogen into fibrin during coagulation. Hirano suggests that the reaction is due to peculiarities of the individual's blood plasma and that there is some intimate relation between salvarsan and its substitutes and the super-renal system in the development of anaphylactic shock because of the fact that adrenalin has been employed successfully in the treatment of the reaction. Kolmer, Schamberg and others have expressed their opinion that these reactions are due 


\section{BRITISH JOURNAL OF VENEREAL DISEASES}

in part to an unidentified impurity in the blood. None of these theories explain satisfactorily the exact nature of this reaction, and it is probable that more than one cause is present in the production of the acute reaction which occurs either during or immediately subsequent to the administration of arsenobenzol intravenously. The reactions, while they may occur subsequent to intramuscular medication, are usually delayed two to three hours when this method of administration is employed and are much less frequent in their incidence.

In our experience vaso-dilator reactions are very uncommon provided the patient is carefully examined prior to the administration of the drug and is carefully prepared ; provided care is exercised in the dissolving of the drug, that it is given in sufficient dilution, that the " 606 " is carefully alkalinised, that both " 606 " and " 9I4" are filtered before use, administered slowly, and that none of the drug is allowed to escape into the tissues. If these conditions are fulfilled the incidence of all immediate reactions, both minor and vaso-dilator phenomena, will be extremely small. Occasionally one meets with a sensitive patient, and it is necessary to consider what steps should be taken to safeguard this type of individual. In every case the preparation of the patient, the preparation of the drug and the technique of administration must be on sound lines. If there is reason to suspect the onset of a reaction, adrenalin chloride, I c.c. intramuscularly, may be used as a prophylactic agent ; for this purpose it is given three or four minutes prior to the intravenous injection. Atropin in doses of not less than one-fiftieth of a grain hypodermically half an hour before the administration of the drug is also a useful prophylactic, and in association with this Stokes has suggested the desensitisation of the patient by giving a previous minimal dose of the drug half an hour prior to the larger therapeutic dose. Stokes is of opinion that the combination of the atropin and desensitisation enables the salvarsan treatment to be continued in patients who had had repeated severe reactions. Personally both these methods have proved as efficient in my hands as adrenalin and are not so liable to cause gross cardiac reactions in the patient. Tyramine has also been recommended in doses of one-third of a grain by Jackson and Smith, and more recently Spiethoff 4 has strongly recommended 50 to 60 drops of baldrian- 


\section{EFFECTS OF ARSENOBENZOL TREATMENT}

dyalisat (Recvalysat's preparation) by mouth prepáratory to the injection one hour later. He states that it has the useful quality along with salvarsan of raising the blood pressure, and if objection is made to the taste of it three tablets of bromural, a preparation of baldrian, may be substituted.

When the actual condition has developed, during or subsequent to any injection, adrenalin chloride intramuscularly, in a dose of I c.c. every four hours, is undoubtedly the most eftective remedy.

In the future therapy of any such cases the adoption of the intramuscular method of treatment will avoid practically all trouble. If intravenous therapy is indicated a change to another drug or desensitisation is often effective in preventing the recurrence of the phenomena.

Of the conditions which arise within the first twentyfour hours, such as herpes, urticaria and albuminuria, none of them are of gross significance with the exception of serous apoplexy or cerebral toxæmia.

This latter is a very severe and often fatal reaction. The others are important in that they are a warning that the patient is highly sensitive to the drug, and their onset should make one go guardedly in the future administration of the drug, both as to its dosage and as to the intervals between successive doses. If these signs occur it is often advisable to temporarily suspend treatment, or at least to reduce the dose and to prolong the intervals between doses. It is also advisable in all such cases to enquire carefully into the condition of the urine, to examine for cardio-vascular changes and to exclude alcoholism and any possible intercurrent focal sepsis.

Serous apoplexy is remarkable in that it generally occurs within twenty-four to forty-eight hours after the second or third injection of the drug, and up to now no satisfactory explanation has been given of its causation. It is frequently fatal unless prompt measures are taken to deal with it. Ehrlich considers that it is due to the toxic action of an oxidation product of salvarsan, and Milian, ${ }^{\mathbf{5}}$ with Ehrlich, that it is due to insufficient quantities of adrenalin in the blood. $\mathrm{McDonagh},{ }^{6}$ on the other hand, expresses the opinion that it is a syphilitic lesion and not due to salvarsan. Friedreich ${ }^{7}$ emphasises the importance of alcohol in producing it because the capillaries are previously damaged by it. Intercurrent disease, and especi- 


\section{BRITISH JOURNAL OF VENEREAL DISEASES}

ally endocrine deficiency, are also mentioned as causal agents. Kolle is of opinion that influenza is a large predisposing factor in the production of encephalitis. Loeb, quoted by Maerz, ${ }^{8}$ also emphasises this in the ætiology. Fortunately its incidence is comparatively rare; that it is so fatal is due to the fact that the reaction comes on late and that in many cases it is mistaken by the physician who is called in to see the patient for a simple apoplexy or uræmia. Kolle, as quoted by Burton Thom, ${ }^{9}$ advocates desensitisation, or, alternately, the administration of 60 c.c. of a 2 per cent. solution of bicarbonate of soda five minutes before the injection to prevent this complication. In our experience there is no known method of prophalyxis, and it is impossible to tell in which case it will develop. Strong, active, healthy individuals are as prone to it as more debilitated subjects. Seven cases, three of which have recovered, have come under my notice ; fortunately all three were seen within a few hours of the onset of the complication. The method of treatment adopted in each case was venesection, lumbar puncture and drainage of the theca till dry, combined with the administration of adrenalin in doses of I c.c. every four hours. Adrenalin restores the diminished elasticity of the arteries and thereby avoids hæmorrhages in the brain. The same methods were adopted in the four fatal cases, but failed to effect any change in the patient. In one of these latter lumbar puncture in three different areas showed the presence of pure blood in the spinal fluid due to a hæmorrhage into the ventricle; in the second the spinal fluid showed an acute pneumococcal meningitis. The cases which recovered are interesting. In the first case the patient was an adult, aged twenty-four, of very active and athletic habits. He was referred to me as a case of acute gonorrhœa, but on examination was found to have a meatal chancre. Successive doses of 0.45 gramme neokharsivan were administered on the first and fifth days, and the third dose, 0.6 gramme, was administered on the twelfth day; neither at or immediately subsequent to the injections did the patient show any sign of intolerance. The day following the third injection the patient was out shooting, and the same evening during dinner collapsed and became unconscious. This was thirty hours subsequent to the injection. The treatment mentioned above was instituted. The patient remained unconscious for 


\section{EFFECTS OF ARSENOBENZOL TREATMENT}

four days, and for three months subsequently he had no recollection of what had happened. The whole picture of what had occurred suddenly dawned on him when a specimen of blood was being taken from his vein three months subsequently with a view to ascertaining the necessity of further treatment. The second case of recovery was one in which silber-salvarsan was being administered to a female patient, aged thirty-eight. She was suffering from disseminated sclerosis, and subsequent to the third injection of 0.2 gramme there occurred a slight and very transient vaso-dilator phenomena ; thirtysix hours later vomiting and diarrhœa set in, and the patient showed considerable irritability and inability to pronounce words. In playing bridge she showed complete inability to play a game at which she had previously been proficient. Vomiting and diarrhœa set in and became gradually worse, and were attributed by her medical attendant to an attack of ptomaine poisoning. In fortyeight hours she became completely unconscious and lost the power of both her sphincters. In this case lumbar puncture, venesection and adrenalin were exhibited. The vomiting ceased immediately after lumbar puncture, the sphincters were under control, partial consciousness was regained in four hours, and complete consciousness twenty-four hours later. The symptoms of disseminated sclerosis were aggravated for some time, as was also the patient's inability to express her thoughts with the appropriate spoken word. The third patient who recovered was a boy, aged twenty, suffering severe pain from Hodgkin's disease. This patient was debilitated and had been under morphine treatment for a very considerable time. Salvarsan was exhibited in an attempt to ease the pain and to influence the progress of the case ; 0.3 gramme of N.A.B. was administered intravenously and twenty-four hours later complete unconsciousness set in. Under the treatment previously mentioned the patient regained consciousness twelve hours later, and it was of interest to note that the acute pain previously associated with his lympho-sarcoma was very favourably influenced for the succeeding two to three weeks.

An alternative method of treatment is advocated by Weil, ${ }^{10}$ who is of opinion that one can compare hæmorrhagic encephalitis with hæmorrhages which occur in the systemic system such as epistaxis and menorrhagia. $\mathrm{He}$ 


\section{BRITISH JOURNAL OF VENEREAL DISEASES}

treats such cases with subcutaneous injections of human blood.

There is no doubt that further intravenous injections of any of the arsenobenzol drugs are absolutely contraindicated in any case which has shown a severe hæmorrhage in the central nervous system, and even intramuscular injections are scarcely justifiable although one had administered them without complications in one of the cases quoted. Intramuscular injections, however, may also be followed by hæmorrhagic encephalitis, and in any case which has shown this gross sign of intolerance the safest and best method of treatment is the use of some other anti-syphilitic drug, such as bismuth, for further treatment.

Later Reactions.-Malaise, general depression and loss of weight may occur at any time in patients being actively treated over long periods by arsenobenzol and its substitutes. These conditions often come on insidiously and, in a resistant case, are in part due to the nature of the infection and in part to the treatment. This is markedly so in cases with a persistent positive Wassermann reaction, where the temptation to treat continuously is great and the patient is necessarily exposed to the risk of cumulative effects from continued intensive treatment. In such cases the method of avoiding these complications is to always remember the fact that one is treating a human being as much as a syphilitic infection. Cumulative effects can be avoided by giving intervals of rest from arsenobenzol therapy, and in those intervals of rest one can quite easily exhibit other anti-syphilitic drugs. Tonic treatment and especially iron is valuable in all such cases. One doubts very much whether the joint exhibition of mercury and arsenic, or of bismuth and arsenic, increases the toxicity of both drugs ; certainly it increases their potency, and I have no hesitation in saying, from experience of a large number of cases, that dual therapy can be exhibited quite safely if the ordinary precautions of watching the body weight, testing the urine and carefully examining the patient clinically from time to time are carried out during the treatment.

Albuminuria.-Albuminuria is occasionally met with as a result of the first or second administration of arsenobenzol or its derivatives. It is a temporary toxic reaction and requires no special treatment. A more difficult con206 


\section{EFFECTS OF ARSENOBENZOL TREATMENT}

dition to deal with is the albuminuria which is met with later on in treatment. Mercury, and to a less extent bismuth, are irritating to the kidneys if they are not carefully watched and the necessary intervals of rest allowed. When used in conjunction with arsenobenzol they may augment the injury. If albuminuria is met with in any case which has been treated by arsenic and mercury or by arsenic and bismuth, it is always advisable to rest the patient from all intensive specific treatment for a time. During this time the routine measures, dietetic, medicinal and others, which are indicated in any acute or sub-acute nephritis, are tried and the amount of albumen present is estimated from day to day. If the albumen disappears one is justified in assuming that it has been due to therapy, and subsequently arsenobenzol alone should be tried in small tentative doses and persevered with if it does not cause a recrudescence of the albuminuria. In other cases the amount of albumen may not decrease on cessation of the specific therapy, and in such it is essential to consider to what extent the condition is due to syphilis. If the albuminuria is syphilitic, tentative gradually increasing small doses of arsenobenzol in association with iodides will tend to lessen the amount of it, and while the dosage given must be guarded it is usually possible by careful watching of the renal function to exhibit arsenic with great advantage to the patient in general and to the renal efficiency in particular. Dual therapy is to be avoided in the future treatment of all such cases, and a more than careful watch on the condition of the urine from week to week is essential.

Dermatitis.-While there is considerable divergence of opinion as to whether the disease or the remedy is the cause of many of the toxic effects which arise during arsenobenzol treatment, it is generally conceded that the arsenical radicle in the arsenobenzol preparations gives rise directly to toxic erythematous rashes which, if severe or untreated, may proceed to exfoliation.

Certain individuals have a special idiosyncrasy of the skin to therapeutic irritants, and while this is so there is undoubtedly a greater tendency to this condition arising in four types of patient :-

(a) Alcoholics.

(b) Those who suffer from an already existing dermatosis.

v.D. 


\section{BRITISH JOURNAL OF VENEREAL DISEASES}

(c) Subjects with septic foci elsewhere in their tissues, and especially oral sepsis.

(d) Highly-strung nervous patients.

Stokes ${ }^{11}$ has drawn special attention to focal sepsis and to previous dermatoses as ætiological factors in its causation. In practically all the patients in whom this exfoliative accident has occurred in my hands during the last six years both these factors have been present.

From the experience of the past ten years I cannot agree with the observations of Wechselmann ${ }^{12}$, Schamberg and others that the joint exhibition of mercury with the arsenobenzols predisposes to its onset. In close on I00,000 injections in Edinburgh, where dual therapy has always been given, the percentage figure of dermatitis is 0.0024 per cent. as compared with 0.016 per cent. in Schamberg's I2,000 injections, where arsenic alone was given. Nor can I agree with Harrison's ${ }^{13}$ observation that exposure to cold is of great ætiological importance. Exposure, however, does predispose to the dermatitis becoming more severe and to complications such as broncho-pneumonia in a subject of exfoliating dermatitis. Neither is excessive dosage essential in its production. The most severe case I have seen occurred in an adult congenital syphilitic patient after so small a dose as 0.I5 N.A.B. Any of the arsenical products may give rise to it, and the fact that it is met with in non-syphilitic patients points to syphilis having no part in its causation. It is much less frequent subsequent to intramuscular therapy than to intravenous. There is no actual proof that it is due to over-oxidation of the tissues by metallic arsenic as suggested by McDonagh. ${ }^{\mathbf{1 4}}$ Initially it begins as a local or generalised erythema, which rapidly becomes vesicular or œedematous, and finally ends in exfoliation. Generally the condition is ushered in by itching of the back of the hands and of the arches of the feet, and occasionally there is a sensation of formication round the mouth; herpes labialis may be present, general erythema and dermatitis soon follow. The condition may occur within twenty-four hours after the injection or it may be delayed for days or weeks. When the erythema becomes generalised there is often an associated conjunctivitis and a laryngo-bronchial catarrh. The face and eyelids become swollen, itching is intense, and the patient feels miserable; the temperature rises from 208 


\section{EFFECTS OF ARSENOBENZOL TREATMENT}

$\mathrm{IOO}^{\circ}$ to $\mathrm{IO}^{\circ} \mathrm{F}$. The erythematous condition quickly becomes vesicular and finally proceeds to desquamation. In some cases there is only a mild systemic reaction, and the exfoliating process is never a true index of the severity of the arsenical poisoning. There may or may not be albuminuria, and considering the severity of the process it is remarkable how seldom there is any evidence of renal trouble. It is noteworthy also how few of the important structures of the body are affected in spite of the gross pathological changes in the skin. The patient makes a slow but uninterrupted recovery unless complications set in. The most dangerous complication is the development of a broncho-pneumonia, and it is on account of this danger that it is advisable to confine all such cases to bed until the temperature reaction has settled and for some time afterwards. Other complications which may arise are peripheral neuritis and possibly jaundice. In some few cases chronic foci of suppuration may commence and become generalised over the skin surface. The prognosis is completely dependent on the onset of complications and especially of broncho-pneumonia, and if this can be avoided complete recovery is the rule. While it is impossible to state in which case dermatitis will arise, in any patient suffering from seborrhœic dermatitis or other generalised skin lesion, in any alcoholic or in a patient with some definite focal sepsis, it is essential to treat these conditions before administering arsenobenzol. If the latter is urgently required the dosage should be small and the greatest care should be exercised in the preparation of the drug and in its administration.

When an erythematous condition occurs subsequent to an injection it is possible in most cases to prevent the development of dermatitis if one sees the patient soon enough. Sulphur and its derivatives are undoubtedly the most powerful remedies we have for this purpose. All purely specific therapy should cease, the patient should be confined to bed and carefully dieted, the bowels must be kept open and the skin made to act with tepid sponging Sodium thiosulphate should be administered daily by intravenous injection, commencing with a dose of 0.3 to 0.45 gramme, and the dosage gradually increased up to I gramme daily. If the erythema is very marked initial venesection is also of value and sulphur may be given freely by mouth, 30 grains twice or thrice daily. This 


\section{BRITISH JOURNAL OF VENEREAL DISEASES}

treatment by thiosulphate of soda, initially suggested by Ravaut, will cut short and cause resolution of the larger number of cases; it is better tolerated, gives rise to less discomfort and gives more consistent results than either intramine or contramine. Many patients, however, are not seen until the erythematous condition has become vesicular or has proceeded to desquamation. When this has occurred rest in bed is essential and complete avoidance of all exposure to cold. In such cases intravenous injections of thiosulphate of soda will lessen the severity of the condition, but it does not in many cases in my experience, even if intensively given, cut short the desquamation process. Sulphur internally is also valuable in such cases, and boro-calamine lotion or plain vaseline locally lessens the itching and protects the inflamed skin. When the temperature has subsided and exfoliation is in progress, hot bran baths assist in removing the desquamated skin, while a boracic starch poultice is the best method of soothing and clearing up the face condition.

Alternative methods of treatment have been recommended by McBride and Denny. ${ }^{15}$ As an adjunct to intravenous sodium thiosulphate they advise giving initially I5 grammes sodium thiosulphate in 480 c.c. of water by mouth, followed subsequently by $\mathrm{I}$ gramme three times daily in solution. Unna recommended the continued administration of adrenalin by mouth as a prophylactic against dermatitis because it tends to obviate the tendency to inflammation of the skin. Kromberger ${ }^{\mathbf{1 6}}$ gives internally " adrenalin syrup," namely, " adrenalin I/I,000 " (5 grammes of it in simple syrup to make 50 c.c.). Four teaspoonfuls of this given daily, that is, 2.5 milligrammes adrenalin, will restore, he states, the patient's tolerance to salvarsan and prevent a recurrence of dermatitis or any idiosyncrasy towards it. Thiosinamin has also been administered intravenously in dermatitis, and Greenbaum ${ }^{17}$ recommends, for its rapid action in all such cases, the dissolving of 3 grains of thiosinamin in 6 c.c. of distilled water to which I or 2 drops of a 2 per cent. solution of glycerine have been added after sterilisation in the autoclave; an average daily dose of 3 grains of the drug is given by Greenbaum.

It is of interest to note that in many cases which develop arsenical dermatitis the Wassermann test, although previously positive, may become negative during the 


\section{EFFECTS OF ARSENOBENZOL TREATMENT}

dermatitis and remain so for some time. I cannot agree with Buschke, Freymann and Bruck, ${ }^{18}$ who state that such cases never show any future manifestations of syphilis, either clinical or serological. Stumpke ${ }^{\mathbf{1 9}}$ found a recurrence of symptoms of syphilis and a positive Wassermann test in seven cases out of eleven who had suffered from arsenical dermatitis. Galliot 20 also quotes cases of serological recurrence and a subsequent meningitic lesion in a subject of dermatitis. In eight out of twenty-four cases of dermatitis which have come under my notice the Wassermann test was only temporarily negative, and in cases of central nervous system infection the condition of the spinal fluid has not in my experience been appreciably altered by the dermatitis.

With regard to the future treatment of those cases, I cannot agree with Ffrench ${ }^{21}$ that treatment may be recommenced as soon as the patient is convalescent ; this may be possible in the simple erythematous cases, but in cases which have proceeded to exfoliation it is more advisable to exhibit some other drug, such as bismuth or mercury to control the syphilis, and to avoid arsenic for six months subsequent to the occurrence of the dermatitis. Nicholas, ${ }^{22}$ Gougerot and others are of opinion that such cases remain intolerant to arsenic for months or years, and that mercury should be relied on for the future treatment. If at any future date arsenical therapy should be necessary, I would strongly advise a change of drug from that which previously caused the dermatitis, the intramuscular method of administration and a very guarded initial dosage. It is advisable in such cases to administer sulphur in some form or other as a prophylactic both prior to and during the exhibition of the arsenical drug.

Jaundice.-Jaundice occurring during and subsequent to treatment by arsenobenzol has given rise to very wide discussion, and there is no general agreement as to its causation. Many consider that it is due to the toxic action of arsenobenzol on the liver tissue. Milian ${ }^{23}$ and others think that the greater number of cases occurring several weeks after arsenical administration are due to the syphilis, and that toxic jaundice is very rarely produced by either " 606 " or " 9r4," and if so that it develops during treatment and not after. More recently there has been a tendency to assume that other factors enter 


\section{BRITISH JOURNAL OF VENEREAL DISEASES}

into the ætiology of jaundice. Icterus occurring during and after salvarsan therapy in many cases shows a striking resemblance to a simple catarrhal jaundice. It is just possible that the increased incidence of jaundice in patients undergoing arsenobenzol treatment, which has been reported from some areas, is due to some extent to an increase in the number of catarrhal cases in the population. The influenza epidemics of the past few years have been to some extent held responsible for this. In our experience jaundice has not been particularly prevalent. When it has occurred it has done so with seasonal variation, and it has in practically every case been particularly mild in its effects. Predisposing factors to its occurrence have undoubtedly been constipation and gastro-intestinal toxæmia. It is interesting to note also that in the opinion of many practising physicians the incidence of catarrhal jaundice has increased of recent years. We are strongly of opinion, therefore, that a very considerable number of the mild cases of jaundice which are met with during and subsequent to salvarsan therapy are not due solely to the salvarsan ; it may be, and no doubt is, a strong contributory factor in its production. Salvarsan is excreted to a great extent through the alimentary canal, and it no doubt irritates the mucous surface of the canal. If, in addition, the gastro-intestinal tract is already in an inflamed condition, due to gastro-intestinal sepsis, it is a simple matter for the inflammatory condition to extend from the intestine through the biliary tract and set up a cholangitis. When it occurs in primary cases with a negative Wassermann, syphilis can certainly be excluded as the cause of it ; it cannot be excluded in other cases, and, as Milian points out, arsenobenzol can be exhibited in many cases of jaundice without any detrimental effect and often with great advantage. We have seen two patients with untreated primary syphilis and a positive blood Wassermann who, on admission to hospital, had acute jaundice which reacted extremely well to careful treatment with arsenobenzol. There are cases, however, in which jaundice may arise in patients who are not subjects of syphilis but are under treatment by arsenobenzol, and in such one must assume that arsenobenzol plays some part in the production of the icterus. Cases have been recorded, for example, in which icterus appeared during the treatment of malaria by salvarsan, and there are 


\section{EFFECTS OF ARSENOBENZOL TREATMENT}

patients who have never had a positive Wassermann who, after prolonged treatment with salvarsan, developed jaundice. The explanation which covers the majority of cases is that there has been during the past five or six years an increase in ordinary catarrhal jaundice and that patients who are subjects of syphilis and who, at the same time, suffer from intestinal toxæmia or are alcoholic, are more prone when subjected to salvarsan treatment to the onset of jaundice. In other words, salvarsan is more likely to injure the liver in the person affected with syphilis who, in addition to syphilis, has intestinal toxæmia and a predisposition to jaundice. One has a parallel in the action of salvarsan on the kidney; salvarsan will rarely damage a healthy kidney; it will, however, precipitate the damage in a kidney which is already diseased and under considerable strain.

Many tests have been evolved to estimate the liver function, with a view to the possible prevention of jaundice and its early detection. The quantity of bilirubin present in the blood is estimated either qualitatively or quantitatively. Any increase is considered as the danger signal of a possible oncoming jaundice. Liver function was studied by the phenoltetrachlorphthalein test by Greenbaum and Brown, ${ }^{24}$ who carried out a series of tests in eighty-six patients who were under prolonged treatment. They found that in 75 per cent. the liver function was normal in patients who had received from fortyfive to I24 intravenous injections, while in patients who had received from one to forty-five injections it was only slightly or not at all altered in 70 per cent. MacCormac and Dodds ${ }^{25}$ found all cases normal in fifty-six patients examined during periods from the time of treatment up to four and a half years afterwards. Gerrard ${ }^{26}$ performed the van der Bergh test in a series of 370 syphilitics undergoing treatment to detect the variation in the bilirubin content of the serum. In $28 \mathrm{I}$ no increase was found at any time, in the remaining 89 it was definitely increased, but six cases only showed an increase of three or more units during treatment, and these Gerrard takes as definitely demonstrating a latent jaundice ; four of these six cases subsequently developed jaundice. Gerrard suggests that the van der Bergh test, if performed as a routine, would strongly safeguard against the development of postsalvarsan jaundice. It is difficult in large series of out- 


\section{BRITISH JOURNAL OF VENEREAL DISEASES}

patients to have this or similar prophylactic tests carried out, but we have been able to have this test done in any case which has developed jaundice with a view to estimating the liver function before recommencing treatment with arsenobenzol compounds. In none of the cases up to now which have been so tested has any loss of liver function been found, and in all such cases there has been an absence of any recurrence of the jaundice although large amounts of the arsenobenzol drugs have been given.

When the condition has developed while the patient is under treatment, or subsequent to treatment, it is always advisable to stop all therapy and to treat the case on similar lines to the treatment of a simple catarrhal jaundice. Rest in bed, evacuation of the bowels, careful dieting, the administration of alkalies in association with salicylate of soda and rhubarb, all assist in improving the condition. Glucose intravenously, 20 c.c. of a 20 per cent. solution given daily, and a diet rich in glucose, should be taken. We have made it a routine practice during the last two and a half years to administer 50 grammes of glucose by mouth, to all patients who are having arsenobenzol therapy, and since doing so we have met with extremely few cases of jaundice. There is no doubt that MacLean's theory, that the fasting which is insisted on prior to the administration of arsenobenzol decreases the glycogenic content of the liver and that, as a result, the liver is more vulnerable to arsenic. The administration to the patient of 50 c.c. of glucose by mouth about half an hour prior to the intravenous injection may, by increasing the glycogenic content of the liver, be of considerable assistance in fortifying the liver against the action of the arsenic. Occasionally one obtains good results in the treatment of a case of established jaundice by ichthyol given internally in capsule in doses of five minims twice or thrice daily.

Since I9I7 we have not met with a case in which acute yellow atrophy has resulted, although the amount of treatment given now is much greater than administered at that time.

When a patient has developed jaundice it is essential before recommencing the therapy to get the excretory apparatus of the body into a healthy condition. It is essential also to rest the patient for at least one month 


\section{EFFECTS OF ARSENOBENZOL TREATMENT}

subsequent to the disappearance of all the symptoms of icterus, and it is advisable to test the liver function before recommencing the treatment by arsenic. It is always safer and more judicious in such cases, after the recovery from jaundice, to initially institute treatment by bismuth or mercury, and only if the patient tolerates these drugs well to commence arsenobenzol therapy. This latter in such cases should be given in small doses frequently rather than in massive doses at longer intervals. In no case which has shown acute atrophy should arsenic subsequently be given.

If one might sum up regarding the prevention of intolerance to arsenobenzol and its substitutes, one would emphasise the necessity for the careful preparation of the patient, for careful examination of the patient prior to each administration, and especially so of his weight and of his urine, for care as to the dosage and the technique of the administration. Avoidance of alcohol must be an essential throughout the whole course of the treatment. When, in addition to these safeguards, one gives, at each administration of the drug, moderate doses at safe intervals and allows at the end of each course rest intervals which are designed to prevent the accumulation of arsenic in the system, many of the later signs of intolerance will not arise.

If giving moderate doses a safe interval between each is one week. The average course of injections should extend over eight or ten weeks, and the average rest interval between courses should be from four to twelve weeks. In the longer intervals other methods of therapy may be exhibited.

Except in the early stages of the disease, heroic therapy by massive doses is not of such value as the more gradual sterilisation of the patient by moderate and safe dosage.

Intramuscular treatment is preferable to intravenous in the later stages because of the more gradual absorption and slower excretion of the drug. The method of giving at frequent intervals small therapeutic doses which the organism can handle has always appealed to me as the safest and most effective procedure in any case with a tendency to intolerance. Above all, in cases with a permanent positive Wassermann, including congenital cases, one must treat the syphilitic patient rather than the positive Wassermann of syphilis. 


\section{BRITISH JOURNAL OF VENEREAL DISEASES}

Much of the material which I have given you is common property, and there are many other questions which I should like to have referred to had time permitted. I must, I feel, crave your indulgence, and confess that I had difficulty in knowing how I could most profitably utilise the time at my disposal to open the discussion on such a very wide subject. I should like before closing to refer in a word to the latest of the arsenical products, namely, tryparsamide. Up to now I have exhibited this drug in twelve cases of central nervous system syphilis with apparently beneficial effect. If one excludes any lesion of the optic tract by careful examination of the eye before commencing treatment, the drug is, I find, extremely well tolerated by almost all patients who have normal fundi. The average dose is 3 grammes weekly. One patient under my care, a previous subject of jaundice, has in the past twelve months had 84 grammes intravenously without showing any single sign or symptom of intolerance. I have had no trouble with blindness or optic neuritis, but I would strongly advise those of you who are tempted to use this drug in central nervous system syphilis to avoid its use in any case in which there is disease of the optic nerve.

\section{REFERENCES.}

(I) Schamberg, Kolmer, Raiziss. Amer. Journ. of Syph., January, I922.

(2) WeIss. Archives of Dermat. and Syph., March, I920.

(3) Stokes, J. H. Archives of Dermat. and Syph., September, I920.

(4) SpIETHOFf. Urologic and Cutaneous Review, p. 97, February, I925.

(5) Milian. Paris Méd., xiv., I97, March Ist, I924.

(6) McDonagh. "Venereal Diseases, their Clinical Aspect and Treatment," p. I3I.

(7) Friedreich. Archives of Dermat. and Syph., Berlin, I42, p. 434, March 2nd, I923.

(8) Maerz. Münch. med. Woch., 70, p. 424, April 6th, I923.

(9) Burton Thom. New York State Jour. Med., 23, p. I55, April, I923.

(IO) WeIl. Presse Méd. Paris, 3I, 657, July 28th, I923.

(II) Stokes, J. H. Med. Clin. North Amer., iii., 82, p. 844, I9I9 ; Archives of Dermat. and Syph., April, I92I, 38, p. 57.

(I2) Wechselmann. Archives of Dermat. and Syph., April, I92I, p. 57 I.

(I3) Harrison, L. W. "The Diagnosis and Treatment of Venereal Disease," p. 387.

(I4) McDonAgh. "Venereal Diseases, their Clinical Aspect and Treatment," p. 225.

(I5) McBride and Denny. Archives of Dermat. and Syph., 7, p. 63, January, I923. 


\section{EFFECTS OF ARSENOBENZOL TREATMENT}

(I6) Kromberger. Wein. klin. Woch., 36, p. 237, March 29th, I923.

(I7) Greenbaum. Jour. Amer. Med. Assocn., 83, p. 38, July 5th, I924.

(I8) Buschke, Freymann and Bruck. Berliner klin. Woch., April I6th, I92I; May I6th, I92I.

(I9) Stumpke. Med. Clin., January, I923.

(20) Galliot. Annales de Maladies Vénériennes, May, I922.

(2I) FFRENCH. Lancet, June I2th, I920, p. I263.

(22) Nicholas, Gougerot. Journ. de méd. de Lyon, April 2oth, I92I, pp. 876-889.

(23) Milian. Paris Méd., xiv., p. I97., March Ist, I924.

(24) Greenbaum and Brown. Journ. Amer. Med. Assocn., 82, p. 88, January I2th, I924.

(25) MacCormac and Dodds. B.M.J., London, p. I200, December 22nd, I923.

(26) Gerrard. B.M.J., London, p. 224, August 9th, I924. 